\title{
Magellan vu par Stefan Zweig. Mémoire d'un voyage/prélude d'un exil
}

\section{Magellan seen by Stefan Zweig. Memory of a Journey / Prelude to an Exile}

\author{
ANA MARIA ALVES \\ Instituto Politécnico de Bragança; \\ Centre de Recherche de Langues, Littératures et Cultures de l'Université d'Aveiro \\ amalves@ipb.pt
}

Mots-clés

mémoires ; voyage ; exil ; intellectuel ; résistance ; traversée.
En 1518, un Portugais exilé du nom de Magellan persuade le roi d'Espagne, Charles Quint, d'un projet ambitieux. Il le convainc de lui donner une flotte afin de « découvrir le passage menant de l'océan Atlantique à l'océan Pacifique, et par là le chemin permettant de faire le tour du monde » (Zweig, 2003 : 178). Cette traversée sera remplie de multiples péripéties : mutineries, froid, faim, maladies, entre autres.

Notre objectif est de remémorer l'histoire d'un grand circumnavigateur portugais par le biais d'une biographie écrite magistralement par Stefan Zweig. Étonné de constater le peu d'informations existant sur Magellan, Stefan Zweig entreprend des recherches et reconstruit l'incroyable destin de ce navigateur, un des aventuriers les plus audacieux de l'époque. Dans une prose typiquement fluide et élégante, il nous emmène dans un fascinant voyage de découverte. Cette biographie, inspirée lors de son voyage vers le Brésil en 1936, traversée entreprise au début des années d'exil alors qu'il fuyait la barbarie nazie, rend finalement justice à Magellan.

In 1518, an exiled Portuguese called Magellan persuaded the King of Spain, Charles V, to sponsor an ambitious project. He convinced the monarch to give him a fleet in order to: "discover the passage leading from the Atlantic Ocean to the Pacific Ocean, and thereby the path to travel around the world"' (Zweig, 2003: 178). This passage proved full of adventure: mutiny, cold, hunger, disease, to name just a few of the challenges.

Our goal is to recall the story of the great Portuguese circumnavigator through Stefan Zweig's masterly written biography. Surprised at the lack of information on Magellan, Stefan Zweig did research on the subject and retraced the incredible destiny of this navigator, one of the most daring adventurers of his time. In a typically flowing and elegant prose, Stefan Zweig takes readers on a fascinating exploration journey.

The writing of this biography, which finally does justice to Magellan, was inspired to the author by his voyage to Brazil in 1936, in the early years of his exile away from Nazi barbarism. 
Nul besoin de se soucier d'argent, de se préparer, de lire des livres, de se mettre en quête d'un logement [...]. En outre, c'est bon marché, pratique, et avant tout facile à coup sûr la formule de l'avenir. On ne voyagera plus. On sera voyagé.

(Zweig, $2000: 137$ )

Stefan Zweig, écrivain, nouvelliste, dramaturge, historien, biographe et Européen cosmopolite né le 28 novembre 1881 à Vienne, en Autriche-Hongrie, a vécu la seconde moitié de son existence dans l'entre-deux guerres.

Issu d'une famille juive autrichienne, croyante mais modérée, fils d'un riche industriel israélite, il suit des études en langues et littératures germaniques et romanes, philosophie et histoire. La situation économique de sa famille lui permet de se cultiver sans aucun souci, voyageant beaucoup en Europe, où il réalise, en 1900, son premier périple en France, son pays de prédilection, tout d'abord en Bretagne et par la suite à Paris. A partir de 1901, il s'engage dans la traduction littéraire d'écrivains français, comme Paul Verlaine et Charles Baudelaire. Il décide de reprendre ses voyages entre 1908 à 1909, et traverse l'Inde, Ceylan, la Malaisie, l'Indochine. Bien avant la Première Guerre Mondiale, il continue sa découverte du monde et part aux États-Unis, aux Antilles, à Cuba, en Jamaïque et à Porto-Rico. Ses incursions lui permettent de prendre contact avec beaucoup d'artistes et intellectuels étrangers.

Entouré d'écrivains de renommée internationale, comme Romain Rolland (qu'il rencontre en 1910), il connaîtra sa future épouse, Friderike Maria von Winternitz, en 1912. Hormis Romain Rolland, il compte parmi ses amis : Sigmund Freud, Emile Verhaëren, Thomas Mann, H.G. Wells, James Joyce, Rainer Maria Rilke, Maxime Gorki, Emile Verhaeren, Paul Claudel, André Gide et Paul Valéry, Albert Einstein, Auguste Rodin, entre autres.

Avant la Première Guerre Mondiale, il jouit d'une notoriété exceptionnelle, tout en menant l'existence rêvée d'un « jeune bourgeois voué au spirituel et au culte de l'art » (Niemetz, 1996 : 96). Dans Le Monde d'Hier, Zweig souligne que sa ville natale, Vienne, « se posait en héritière de tout le passé prestigieux de l'humanité et en premier lieu de l'Antiquité » (Zweig, 1993: 49). Cette ville, excitante et magnifique, se dressait comme carrefour des échanges interculturels, comme centre intellectuel où régnait la culture humaniste, et où, d'après lui, « chaque bourgeois [...] était promu par son éducation à ce cosmopolitisme qui répudie tout nationalisme étroit, à la dignité, enfin, de citoyen du monde » (Zweig, 1993 : 42). Pacifiste convaincu, il s'engage dans l'armée autrichienne en 1914, et son action sera toujours celle d'un écrivain actif, luttant pour une unification de l'Europe face à la montée du nazisme en Allemagne.

Après la Première Guerre Mondiale, il parcourt à nouveau l'Europe, de 1916 à 1933, reprenant contact avec différents amis intellectuels dont il avait été séparé durant la guerre et tient diverses conférences dans lesquelles il prône la fraternisation plutôt que les conflits. Zweig est convaincu qu'il doit se positionner en intermédiaire entre hommes et nations; c'est pourquoi il trouve son succès « en diffusant par sa vie et ses écrits un message d'amitié, de tolérance et de compréhension internationale » (Kiser, 1998 : 59). Fidèle à ses idéaux, il milite pour la liberté en Europe et prévient ses auditeurs des périls de l'extrémisme politique.

Dès 1933, ce monde, cet âge d'or dont il était l'incarnation, se voit ébranler par l'arrivée au pouvoir d'Adolphe Hitler. L'obscurité que Zweig appréhendait tant se propage rapidement sur l'Europe. Les premiers autodafés se déroulent à Berlin, où ses livres sont brûlés. Zweig sent que les forces dévastatrices de la Première Guerre Mondiale apparaissent pires encore lors de la montée du nazisme au pouvoir. L'interdiction de la publication de ses livres en Allemagne et en Autriche marque l'écrivain à jamais. Il se sent dépossédé de son identité, de ses convictions 
humanistes, comme il le souligne d'ailleurs dans Le Monde d'Hier: " D'un trait de plume, on avait transformé le sens de toute une vie, une vie en un non-sens ; j’écrivais, je pensais toujours en langue allemande, mais chaque pensée, chaque vœu que je formais appartenaient aux pays qui étaient sous les armes pour la liberté du monde »(Zweig, 1993: 505). Ravagé par ses attaques, il émigre en Angleterre (Bath, puis Londres) à la fin de 1933, marquant, de la sorte, son opposition au régime national-socialiste et fuyant une Autriche où la littérature, avait, d'après Josef Strelka, «cessé d'exister » (apud Kreissler, 1980 : 227). De l'Angleterre, il reprend ses voyages - à travers l'Europe et l'Amérique du Sud. Il visitera, à plusieurs reprises, de nombreux amis exilés à Sanary-sur-Mer, la célèbre capitale de la Littérature Allemande, comme la décrit Ludwig Marcuse, ami de Zweig.

En 1936, plus précisément le 8 août, il fuit l'Europe pour se rendre au Brésil, où les périls augmentent. Pendant le trajet, « la traversée est agréable, reposante et instructive. Il lit des livres en espagnol [...] et un ouvrage sur Magellan qui lui suggère l'idée de consacrer au navigateur une miniature historique ou un roman (Niémetz, 1996 : 598). L'idée se concrétise, il entreprend la rédaction d'une biographie dédiée à l'explorateur et navigateur Magellan, «né à Sabrosa, dans la province de Tras-os-Montes, au Portugal, vers 1420 [issu] d'une famille distinguée, des meilleures, et des plus anciennes du royaume [comme il l'écrit lui-même] dans son testament du 17 décembre 1504 »(Pigafetta, 1991: 46). En utilisant une base documentaire solide, Zweig rend hommage au navigateur portugais qui a œuvré pour le progrès. Dans la préface de la biographie, Zweig montre les traits de Magellan, qu'il considère extraordinaire, avouant que «dans les grands faits de l'histoire, il y a toujours, parce qu'ils s'élèvent tellement au-dessus de la commune mesure, quelque chose d'incompréhensible; mais ce n'est que grâce aux exploits incroyables qu'elle accomplit que l'humanité retrouve sa foi en soi » (Zweig, 2003 : 14).

Tout au long de cette biographie romancée, Zweig dresse un tableau prodigieux de l'époque et nous invite à repenser l'histoire. Il expose la vie du navigateur portugais, tout en nous transportant dans l'Epoque des Grandes Découvertes maritimes de Christophe Colomb et Vasco de Gama, dans un espace où les Portugais et les Espagnols s'étaient vu accordés, par une bulle papale du 4 mai 1493, les terres à découvrir et, en 1494, par le traité de Tordesillas, le partage des découvertes outre-Atlantique. D'après ce compromis hispano-portugais, signé le 7 juin 1494 , l'est du $50^{\mathrm{e}}$ méridien sera portugais et toutes les découvertes à l'ouest appartiendront à la couronne espagnole.

Ces deux royaumes ibériques recherchaient une supériorité commerciale afin de déployer leur empire colonial. Des concours de circonstances ont projeté, à l'âge de 39 ans, Magellan dans cette compétition, plus précisément le 20 septembre 1519 à l'aube, et sous la bannière espagnole ce navigateur commença une formidable odyssée, "le plus long voyage de découvertes, l'aventure la plus hardie de l'histoire » (131). Cet homme «s'est donné pour tâche de découvrir le passage menant de l'océan Atlantique à l'océan Pacifique, et par là le chemin permettant de faire le tour du monde » (178), avec une flotte qu'il « conduit le long de la côte de l'Amérique du Sud plus loin que ne l'a jamais fait aucun autre navigateur » (178). Il part donc avec une flotte de cinq navires chargés de vivres abondants pour nourrir un équipage de 265 hommes :

L'alpha et l'oméga de toute nourriture, c'est le biscuit du marin : Magellan en a fait embarquer 21380 livres, qui coûtent, avec les sacs, 372510 maravédis. [...] A côté des sacs de farine, de haricots, de lentilles, de riz et autres légumes secs se trouvent 5700 livres de porc salé, 200 tonneaux de sardines, 984 fromages, 450 cordons d'ail et d'oignons. [...] Afin de 
maintenir en bon état le moral de l'équipage Magellan a fait acheter le meilleur Xérès : pas moins de 417 [...] et de 253 tonneaux. (118-119)

Zweig nous convie à cette expédition qui nous montre un Magellan intrépide mais minutieux, qui est décidé à trouver les soutiens nécessaires pour cette aventure. Pour un tel projet, il demandera le soutien de l'Espagne, auprès de l'empereur Charles Quint, et non de sa patrie, pour laquelle il avait dédié " dix années de service dévoué » (89). En effet, après ses campagnes des Indes et du Maroc, il s'était vu refuser " une pension élevée » (63) et une " "moradia" symbol[e] [de] dignité occupée par un gentilhomme dans la maison du roi » (66). Cette demande avait été faite au Roi Manoel, que sa chance a fait surnommer "el fortunado" qui n'avait, en aucun cas, apprécié la requête de Magellan qui avait été faite d'une «voie franche et directe, la pire qui soit à la cour » (66). Le navigateur se voyait encore une fois repoussé par son roi, qui l'avait déjà accusé auparavant de complicité avec les Maures, à qui il avait, soidisant, "vendu une partie du butin » (63) résultat de la vente de bétail qu’il avait été chargé de surveiller. Dû à cet incident, Magellan avait vu « son honneur entaché » (63) et avait été accusé de «prévarication envers l'État» (63). Face à cette accusation, Magellan embarque pour le Portugal pour avoir une audience avec le roi, dans le but de se défendre de cette inculpation. Le roi, qui vient d'« être informé par le grand quartier général d'Afrique que cet officier insubordonné est parti du Maroc de sa propre autorité, sans avoir sollicité de permission [...] agit envers Magellan comme à l'égard d'un vulgaire déserteur [...] [et] lui intime [...] l'ordre de rejoindre, sur-le-champ, son poste en Afrique » (64). Après un retour obligatoire à Azamor, Magellan reprend son élan pour quémander son droit de pension auprès du roi et pour lui parler de son projet maritime mais, malheureusement, celui-ci est à nouveau repoussé. Le roi Manoel ne prévoyait pas « qu'avant peu il payerait de milliers de ducats d'or l'économie de ce demi-cruzado » (67). Après cet épisode, dans lequel Magellan est à nouveau traité comme un déserteur par le roi Manoel, le navigateur décide de prendre le chemin de l'Espagne, où il s'exile, tout en demandant à l'empereur Charles Quint l'autorisation d'affréter une flotte, afin de trouver le chemin des «îles des épices ». Son projet de voyage est alors présenté, lors d'une séance mémorable, devant le Conseil de la Couronne Espagnole. À ce propos, Zweig souligne que Magellan a :

immédiatement frappé les conseillers du roi. Ces derniers se rendent compte que le capitaine portugais n'est pas un de ces esprits chimériques qui depuis le succès de Christophe Colomb bombardent la cour d'Espagne de toutes sortes de projets plus ou moins fantaisistes. Cet homme est effectivement allé plus loin en Orient qu'aucun autre navigateur et lorsqu'il parle des îles des épices, de leur situation géographique, de leurs conditions climatériques et de leur richesse incalculable, ses informations s'avèrent $[\ldots]$ plus sûres que celles que contiennent les archivent espagnoles. (91)

Tout au long de son exposition devant la cour, Magellan se montre convaincu que la route vers l'est ne doit pas être prise, car comme il « l'a montré les îles des épices sont situées tellement à l'est des Indes que ce serait un détour superflu que de vouloir, comme les Portugais, les atteindre par l'est, en contournant l'Afrique, puis en contournant tout l'océan Indien et l'archipel de la Sonde » (92). Le voyage par l'ouest s'avère, d'après lui, beaucoup plus sûr et le conduira aux Moluques. 
D’après Zweig, Magellan lance sa dernière carte pour convaincre son audience lorsqu'il affirme être persuadé qu'il faudra contourner le continent américain pour arriver à son but, cela dit, ouvrir la route des Indes par l'ouest. Pour cela, il décide de se mettre au service de la couronne, révélant qu'il est en possession d'un secret, d'« un passage, [d'] un détroit » (92) reliant les deux océans, qui le mènera à bon port. Zweig nous révèle que Magellan « ne se contente pas de dire modestement comme les autres : j'espère trouver quelque part cette route, il déclare avec énergie qu'il sait où elle se tient» (75).

Zweig nous transmet que « ce ne sont pas les humanistes, les savants qui s'enthousiasment pour ce voyage autour du monde qui doit fixer définitivement les dimensions de la terre et confondre tous les atlas jusqu'alors existants, mais le sceptique Fonseca » (93). Il s'agit du Cardinal Fonseca, l'évêque de Burgos, qui 30 ans auparavant s'était opposé «à l'entreprise de Christophe Colomb» (93). Ce dernier se sent séduit par les paroles de Magellan, et soutient le navigateur devant le Conseil du roi, qui comprend alors que ce projet fera du souverain espagnol " le prince le plus riche du monde ", et permettra par ailleurs de " devancer les Portugais qui étendent déjà leurs mains avides vers ce trésor » (92).

La décision est évidente; Magellan réussit à obtenir l'aide de l'Espagne qui lui remet « une flotte et son équipement d'une valeur totale de huit millions de maravédis » (120). Les cinq navires qui se lancent dans l'inconnu ne " doivent pas rapporter uniquement des résultats cosmographiques, mais aussi de l'argent, le plus d'argent possible à ceux qui ont payé les frais de l'entreprise » (121). Ainsi, Magellan se lance dans la préparation de l'expédition, qui ne semble pas l'effrayer, et prend le contrôle de son vaisseau Trinitad, le navire amiral, qui sera suivi de « quatre capitaines [...] qu'on lui a adjoints en qualité de commandants des autres navires » (123). Juan de Cartagena prend le commandement du San Antonio, Luis de Mendoza celui de la Victoria, Gaspar de Quesada prend la Concepción et finalement João Serrão, le seul commandant portugais, prend à sa charge le Santiago.

Zweig, qui vient de nous plonger dans l'ambiance géopolitique du XVIème siècle tout en exhortant les valeurs humanistes qui sont pour lui un apanage de la compréhension fraternelle entre tous les peuples, met en avant les qualités de ce héros, qui se construit une expérience comme soldat et marin lors de ses premiers voyages dans les mers du Sud.

Tout au long de cette biographie, Zweig brosse l'action et la prouesse de son héros « calculateur, précis, psychologue, et réaliste » (84), soulignant sa destinée en affirmant «ce n'est jamais l'utilité d'une action qui en fait la valeur morale. Seul enrichit l'humanité, d'une façon durable, celui qui en accroît les connaissances et en renforce la conscience créatrice » (Zweig, 2003 : 268).

Dès le début de la narration de cette fascinante épopée, Zweig plante le décor et nous enlace par des arômes d'épiceries : «l'étrange et mystérieux parfum de ces fleurs orientales a grisé de sa magie l'âme de L'Europe »(Zweig, 2003: 19). Comme s'il voulait éveiller les consciences, il nous rappelle la valeur économique de ce bien si précieux qui « valait son pesant d'argent » (19) et ajoute qu'au «XVI ${ }^{\text {ème }}$ siècle, le moindre sac de poivre vaut infiniment plus qu'une vie humaine» (22). En fait, dit-il, le poivre

présentait une telle stabilité monétaire que beaucoup d'états et de villes comptaient avec lui comme avec un métal précieux; il permettait d'acquérir des terres, de payer une dot, d'acheter un droit de bourgeoisie; [...] Par ailleurs, le gingembre, l'écorce d'orange et le camphre se pesaient sur des balances d'apothicaire et de joailler, opération qui se pratiquait 
portes et fenêtres soigneusement closes, de crainte qu'un courant d'air n'emporta une parcelle de la précieuse poudre. (Zweig, 2003 : 19)

Pour plus que cette surévaluation puisse nous paraitre absurde de nos jours, il fallait, à l'époque, prendre en compte les risques, les obstacles, les dangers lors du transport de ce bien si précieux. La marchandise, nous rappelle l'auteur, « doit passer par une infinité de mains avant d'atteindre par-delà les mers et les déserts le dernier acheteur, le consommateur » (Zweig, 2003 : 20).

Tout au long de ce parcours de remémoration, Zweig nous pousse, à un exercice de réflexion critique sur l'histoire.

Les croisades ne sont pas simplement (comme les esprits romantiques les ont souvent dépeintes) une tentative mystico-religieuse d'arracher les lieux saints aux infidèles; cette première coalition européo-chrétienne représente aussi le premier effort logique et conscient ayant pour but de briser la barrière qui ferme l'accès de la Mer Rouge et d'ouvrir les marchés orientaux à l'Europe, à la chrétienté. L'entreprise ayant échoué, l'Égypte n'ayant pu être enlevée aux musulmans et l'Islam continuant d'occuper la route des Indes, il fallait nécessairement que s'éveillât le désir de trouver un nouveau chemin, libre, indépendant. L'intrépidité qui poussa Colomb vers l'Ouest, Bartholomeu Diaz et Vasco de Gama vers le Sud, Cabot vers le Nord, vers le Labrador, est née avant tout de l'ardente volonté de découvrir des voies maritimes franches de toute servitude et d'abattre en même temps l’insolente hégémonie de l'Islam. (Zweig, 2003 : 23)

La biographie que Zweig fait de Magellan, navigateur intrépide, montre combien il est fasciné, captivé par cette personnalité historique et par cette aventure périlleuse qui durera trois ans. Charmé par cet homme qui a travaillé pour le progrès de l'histoire, cet écrivain autrichien transforme sa biographie dans un récit absolument prenant, dans un véritable témoignage historique où l'empathie qu'il ressent envers Magellan se révèle tout au long de ses pages.

L'expédition, dont Magellan est responsable souffrira des revers. Le fait « qu’il ait tout au moins réussi à tourner le rescrit royal et à introduire dans la flotte, malgré les protestations de la Casa de Contratación, trente Portugais, parmi lesquels des parents et une poignée d'amis dévoués » (123) provoquera des rivalités. Jaloux de ce choix, « les capitaines espagnols qu’on lui a adjoints en qualité de commandants des autres navires » (123) et qui lui ont « solennellement juré obéissance et fidélité » (123) alimenteront le malaise au cœur de leur équipage. Jaloux encore car Magellan ne les convoque pas dans son vaisseau afin de leur dévoiler son secret à l'aide de ses cartes. Enragés surtout car

la direction de la flotte ne cesse d'être entre les mains sévères et résolues d'un seul homme et que ce Portugais inabordable et silencieux, obstiné dans son secret, les fait mettre en rang chaque jour comme des recrues et les renvoie ensuite après leur avoir communiqué ses ordres, comme s'ils étaient de simples matelots, ce fait indispose fortement les capitaines des autres navires (135).

Cette attitude de Magellan entraînera les capitaines à de vigoureuses mutineries, auxquelles l'amiral survivra. Ce long voyage sera une épreuve pour la plupart de ses participants qui devront faire face aux tempêtes, aux maladies, à l'épuisement, au froid, à la faim. Certains 
trouveront dans la désertion la solution qui les éloignera de ces cinq embarcations dans lesquelles la vie devenait impossible. Les erreurs cartographiques faites tout au long de cette interminable traversée ne seront pas suffisantes pour arrêter la détermination et la foi inébranlables de ce célèbre navigateur, qui finira par accomplir sa glorieuse prouesse trouvant le détroit considéré comme une voie maritime dangereuse, qu'il " dénomme le canal de la Toussaint, mais que la postérité reconnaissante appellera le détroit de Magellan » (184). La sortie du canal sera découverte quelques jours plus tard ; ainsi Magellan « a tenu la promesse faite à l'empereur. Ce à quoi des milliers d'autres avant lui n'avaient fait que penser, il l'a réalisé : il a trouvé le chemin menant à l'autre mer »(190). Zweig défend à propos de cet exploit, que Magellan :

dépasse tous ceux de son époque. [...] L'exploit de Magellan a prouvé, une fois de plus, qu'une idée animée par le génie et portée par la passion est plus forte que tous les éléments réunis et que toujours un homme, avec sa petite vie périssable, peut faire de ce qui a paru un rêve à des centaines de générations une réalité et une vérité impérissables. (267)

En hommage à Magellan, ce Détroit portera son nom. Cependant, le cap de BonneEspérance restera pour la majorité des navigateurs le passage le plus sûr. Zweig souligne à ce sujet que ce détroit

sera si dédaigné, tombera tellement dans la légende, que cinquante ans plus tard le hardi pirate Francis Drake pourra l'utiliser comme la plus sûre des cachettes, d'où il s'élancera à l'improviste, tel un oiseau de proie, sur les colonies espagnoles de la côte ouest ou sur les transports chargés de métal rare. (284)

Après cette découverte, Magellan « a gagné à la couronne de Castille de nouvelles îles d'une très grande richesse, à l'Église d'innombrables âmes de païns, et tout cela sans verser une seule goutte de sang » (224). Zweig nous confie que, d'après lui, «Dieu est venu en aide à celui qui s'est confié à lui. Il l'a sauvé de dangers tels qu'aucun homme n'en a connu de semblables » (224). Dès ce jour, ajoute Zweig, «Magellan se sent pénétré d’un sentiment de sécurité quasi religieux. [...] Et c'est justement cette croyance qui causera sa perte » (224). En effet, ce sentiment de sécurité lui fera baisser la garde, cessant, de la sorte, d'être aussi prévoyant et prudent comme il l'avait été jusqu'alors. Ainsi, il se laissa entraîner dans un combat auprès d'indigènes, qui n'avaient pas voulu, de façon pacifique, devenir alliés de l'Espagne, et trouva la mort aux Philippines, aux mains de ses sauvages.

Des deux cent soixante-cinq hommes embarqués dans cette expédition, seulement dix-huit reviendront de cet épuisant voyage le 6 septembre 1522. Ils retrouveront l'Espagne à bord du seul navire qui ait survécu à cette expédition - La Victoria, au commandement de Sébastian Del Cano. Ce capitaine espagnol reçoit l'ordre de se présenter «à la cour avec deux de ses hommes, les plus autorisés et les plus intelligents et de lui apporter tous les documents qui ont été rédigés tout au long du voyage » (261).

D’après Zweig, Del Cano, accompagné de Pigafetta et du pilote Alvaro, se présente devant l'empereur. L'attitude du capitaine est dès le début très « suspecte, car il n'a pas transmis une seule ligne de la main de Magellan [...] selon toute vraisemblance une main malhonnête a dû [...] détruire » (262) son journal. Non moins étrange est la disparition du manuscrit du chroniqueur recommandé à Magellan par Charles Quint, Antonio Pigafetta. Ce jeune italien « 
calme et modeste, $[\ldots]$ pris $[\ldots]$ du désir ardent de voir $[\ldots]$ des choses terribles et grandioses de l'océan » (125) se trouvait à bord du navire amiral lors du voyage et, comme le souligne Zweig, était devenu pour « Magellan le membre le plus important de son expédition » (125). Le journal de Pigafetta avait été rapporté personnellement à l'empereur et avait également mystérieusement disparu.

Comme le témoigne Zweig, on voulait manifestement « laisser dans l'ombre tout ce qui avait trait à la résistance opposée par les officiers espagnols au portugais Magellan, afin de pouvoir mieux mettre en lumière le triomphe de Del Cano » (262). Zweig souligne que Pigafetta réagit très mal à la position prise par ce capitaine espagnol. Ce jeune chroniqueur est, à ce moment-là, convaincu que "le monde ne récompense que ceux qui ont la chance d'achever une œuvre, il oublie ceux qui l'ont commencé » (263). Il est en effet révoltant de voir que cet officier espagnol, comme le décrit si bien Zweig, qui « récolte toute la gloire et le profit de l'entreprise est précisément celui qui, au moment décisif, voulait l'empêcher » (263). Celui qui a œuvré pour la mutinerie est récompensé et « on lui accorde une pension viagère de cinq cents florins. [Plus tard] Charles Quint l'élève [...] au rang de chevalier et lui confère des armes qui le désignent nettement comme l'auteur de l'exploit immortel» (263). Plus choquant est la récompense attribuée à Estevão Gomez, le déserteur « qui a abandonné Magellan dans le détroit même et qui est venu déclarer, devant le tribunal de Séville, qu'on n’avait trouvé qu'une baie» (263). Celui-ci « est anobli lui aussi pour avoir, en qualité de guide et de premier pilote, découvert le passage » (263).

Révolté devant tant d'injustice, Pigafetta se retire de l'audience. Celui qui avait précieusement fait la description de cette épopée qui illustrait le premier voyage autour du monde au milieu de ces navigateurs était accablé devant tous les rapports présentés à l'empereur. Ce jeune Italien, " étrange idéaliste », ne s'était pas lancé dans cette odyssée " pour la gloire de l'argent mais pour un amour sincère du voyage, pour la simple joie de voir, d'apprendre et d'admirer » (125); il ne pouvait donc pas confirmer le discours de Del Cano qui, voulait absolument faire silence sur la prouesse de Magellan. C'est Pigafetta qui « fera connaitre à la postérité l'exploit » (125) du navigateur qui a découvert un océan inconnu - le Pacifique -, qu'il traversa, atteignant l'Asie par le Détroit, et prouvant, de la sorte, que la Terre est ronde.

D’après Zweig, si Pigafetta ne peut prendre la parole pour le moment c'est par ce qu'il «se réserve, pour l'amour de la justice, de rendre hommage devant la postérité à l'homme qui l'a accompli. Pas une fois dans son récit du voyage de retour il ne mentionne le nom de Del Cano. [...] Avec une fidélité émouvante Pigafetta se range au côté du vaincu et apporte un témoignage éloquent à celui qui n'est plus » (264). Aujourd'hui encore, comme le souligne Vincent Barros, dans son article publié dans le Courrier international, à l'aube des commémorations des 500 ans de cette célèbre expédition, «le navigateur portugais reste pour l'éternité l'artisan du premier tour du monde, [cependant] c'est bien l'Espagnol El Cano qui l'a achevé. Leurs pays respectifs [...] s'en disputent encore la gloire» (Barros, 2019).

Ainsi, l'histoire de Magellan reste polémique après des siècles. Zweig témoigne d'une grande lucidité quand il nous fait part de cette biographie, écrite de façon passionnante et qu'il a si bien documentée, à partir de recherches personnelles, mais aussi et surtout à partir du récit de voyage de Pigafetta. Convaincu de l'hérö̈sme de Magellan, Pigafetta a rendu justice au premier circumnavigateur qui, avec un courage incroyable, s'est enfoncé vers l'inconnu, non pour une reconnaissance personnelle, mais pour un intérêt universel. Narrant, à son tour, avec précision, toutes les étapes de ce voyage épique, Stefan Zweig rend hommage à un homme avec qui il ressent plusieurs affinités et sur lequel il dépose, lui aussi, le constat d'une grande 
humanité. Zweig est alors convaincu que « la somme des obstacles qu'un homme surmonte en pareil cas donne toujours la mesure véritable, exacte, de l'œuvre et de celui qui l'a accomplie » (101).

La plus importantes des affinités entre l'écrivain et le héros qu'il évoque, a comme sujet la mort emportée par la barbarie.

Après le voyage au Brésil, l'horreur, s'installe dans la vie de Zweig, au temps même où il commence la rédaction de la biographie de Magellan. En effet, après son retour à Londres, il se voit dépossédé, en mars 1938, de sa nationalité et devient réfugié politique après l'invasion de l'Autriche par Hitler. Pour préserver sa liberté de pensée, il se voit obligé à quitter définitivement l'Angleterre et tente de gagner les Etats-Unis, où il pense se fixer. Il se rend en France, à Sanary-sur-Mer. Il y retrouve un groupe d'intellectuels autrichiens qui, comme lui, avaient été obligés de prendre le chemin de l'exil. Ce refuge était également celui de l'intelligentsia allemande, qui s'était opposée au régime du III Reich, et avec laquelle il avait des relations amicales. Le 15 août 1941, il s'embarque pour le Brésil et s'établit à Pétropolis, où il espère encore retrouver la paix de l'esprit, qui a été secouée par les évènements politiques récents :

Je n'ai plus aucune volonté. Je sais que jamais cette existence ne se remettra en place, une vie avec une France détruite, dans une Angleterre hostile - à l'Allemand ou au Juif que je suis - n'a plus de sens, de même, sur le plan littéraire, tout ce que je pourrais entreprendre est paralysé pour des années par le manque de concentration. (Zweig, 1986 : 297)

Il y rédigera son autobiographie - Le Monde d'Hier - œuvre qui montre combien Zweig se sent dépossédé de toute force face à l'échec des idéaux d'un monde dans lequel il s'était posé comme médiateur entre les hommes : il « plaidait pour une communauté universelle d'individus libres » et œuvrait " à l'accomplissement éthique de l'humanité en se fondant sur l'héritage durable des siècles et en respectant toujours et partout les droits et les intérêts les plus profonds de tous » (Niémetz, 1996 : 562). L'expérience de cet échec le fait témoigner : « une fois de plus je dus reconnaitre combien notre imagination humaine est insuffisante et que l'on ne comprend vraiment les sentiments les plus importants, justement, que quand on les a éprouvés en soimême » (Zweig, 1993 : 499). Désespéré par la barbarie qui envahissait l'Europe et la planète entière, il mit un terme à ses jours le 22 février 1942, laissant derrière lui, tout comme son héros Magellan, un monde en manque d'humanité.

\section{BIBLIOGRAPHIE :}

BARROS, Vincent (2019). Magellan divise encore le Portugal et l'Espagne, cinq siècles plus tard. Courrier International, 15-02-2019. https://www.courrierinternational.com/revue-depresse/histoire-magellan-divise-encore-le-portugal-et-lespagne-cinq-siecles-plus-tard (consulté le 9 avril 2019).

KISER, John (1998). La mort de Stefan Zweig, Mort d'un homme moderne. Toulouse : Presses universitaires du Mirail.

KREISSLER, Félix (1980). La prise de conscience de la nation autrichienne, 1938-1945-1978. Tome 1. Paris : PUF.

NIEMETZ Serge (1996). Stefan Zweig, Le Voyageur et ses mondes. Paris : Belfond.

PIGAFETTAA, António (1991). Relation du premier voyage autour du monde de Magellan (15191522). Paris : Édition Tallandier. 
ZWEIG, Stefan (1986). Carnet de notes de la guerre 1940, 22 mai - 19 juin 1940. In Journaux, 1912-1940. Paris : Belfond.

ZWEIG, Stefan (1993). Le Monde d'Hier. Souvenirs d'un Européen. Traduction de Serge NIÉMETZ. Paris : Belfond.

ZWEIG, Stefan (2003). Magellan. Version française par Alzir HELLA. Paris : Grasset. 